and quiescence inflammatory bowel disease (IBD) comparing to controls.

Consecutive children with quiescence IBD, IBS and aged and sex matched healthy controls (HC) were referred for the evaluation of dysautonomia (IBD:

$\mathrm{N}=24$, mean age 15.7 yrs, 16 females; IBS: $\mathrm{N}=18$, mean age 14.8 yrs, 9 females; HC: $\mathrm{N}=18$, mean age 14.2 yrs, 9 females). Dysautonomia was evaluated subjectively with the Composite Autonomic Symptom Score (COMPASS 31), and objectively with the following autonomic tests: heart rate (HR) and blood pressure (BP) responses to the Valsalva maneuver, heart rate response to deep breathing (RSA), blood pressure response to passive tilt, and quantitative sudomotor axon reflex test (QSART). Additionally, heart rate variability (HRV) analysis was performed by Kubios HRV 2.2. Following HRV parameters were compared between the groups in supine and tilted positions: total power of low (LF) and high frequency domain components (HF), normalized HF (HFnu), low-to-high frequency ratio ( $\mathrm{LF} / \mathrm{HF})$, standard deviation of normal-to-normal intervals (SDNN) and percentage of successive RR intervals that differ by more than $50 \mathrm{~ms}$ (PNN50).

Children with IBS scored highest on COMPASS-31, followed by patients with IBD and HC (median 15.6, 8.7 and 2.3 , respectively, $\mathrm{p}<0.001$ ). Similar differences were observed in the orthostatic intolerance and gastrointestinal domains of the COMPASS-31. No differences between groups were observed in HR and BP responses to the Valsalva maneuver, RSA and BP response to passive tilt. Children with IBS had higher sweat volumes on proximal lower leg on QSART (median IBD 0.9, IBS 1.5, HC $0.8 \mu \mathrm{L} ; \mathrm{p}=0.039$ ).

There was no difference in the HRV parameters between groups. However, children with IBS had significantly higher drop in LF $(p=0.01)$ and SDNN

$(p=0.03)$ and lowest drop in PNN50 $(p=0.01)$ during tilt test compared to children with IBD and HC.

We found significant subjective and objective ANS abnormalities in children with IBS compared to children with IBD and $\mathrm{HC}$.

\section{INCIDENCE AND GEOGRAPHICAL VARIABILITY OF PEDIATRIC INFLAMMATORY BOWEL DISEASE IN CROATIA: DATA FROM THE CROATIAN NATIONAL REGISTRY}

Lana Ivković*, Iva Hojsak, Ivana Trivić, Sara Sila, Pero Hrabač, Vlatka Konjik, Irena SenečićČala, Goran Palčevski, Ranka Despot, Orjena Žaja, Sanja Kolaček. Children's Hospital Srebrnjak

\subsection{6/archdischild-2021-europaediatrics.228}

Primary aims of this study were to determine the annual incidence and geographic distribution of pediatric inflammatory bowel disease (IBD), respectively existence of north to south gradient in Croatia as well as baseline characteristics at the time of diagnosis. Secondary aim was to compare data on frequency and distribution of pediatric IBD in two consecutive years.

This is a prospective, cohort, multicenter observational study based on the data obtained from the newly established Croatian national registry for children with inflammatory bowel disease. Children and adolescents younger than 18 years diagnosed with IBD, according to revised Porto criteria, in time period between June 1st, 2016 and May 31st, 2017 were recruited. In order to validate obtained data and to monitor trends in the incidence of the disease, the data were also gathered for the period from June 1st, 2017 to May 31st, 2018.

In season 2016/2017, 51 new cases of pediatric IBD were identified; 19

(37.3\%) Crohn's disease (CD), 28 (54.9\%) ulcerative colitis (UC) and 8

(7.8\%) IBD unclassified (IBD-U). Male preponderance of all three types of the disease was noticed (CD 52.6\%, UC $60.7 \%$, IBD-U 75\%). The median age at diagnosis was 14.8 years; 15.3 for CD, 13.9 for UC, and 14.5 years for IBD-U. Female patients were slightly older than males, but without statistically significant difference $(p=0.279)$. With 723,552 children younger than 18 years in Croatia in 2017 , the annual incidence of pediatric IBD per 100,000 persons per year was 7.05 (2.62 for CD, 3.87 of UC and 0.55 for IBD-U). The lowest incidence was found in Dubrovnik-Neretva and SplitDalmatia County (4.50-4.85/100,000 children up to 18 years of age/year), and the highest was found in Meðimurje County (22.80/100,000 children up to 18 years of age/year). Existence of north to south gradient was observed with almost two times higher incidence in northern region compared with southern parts of the country $(8.38 / 100,000$ vs. $4.26 /$ 100,000).

Data from consecutive season (2017/2018) identified 50 new cases of pediatric IBD, with higher proportion of CD (44\%) compared to year before.

The incidence of pediatric IBD in Croatia is 7.05 per 100,000 persons younger than 18 years per year, with significant decreasing trend in incidences between northern and southern regions of the country.

\section{IBD PHENOTYPE ON DISEASE PRESENTATION AND EARLY DISEASE-COURSE IN PEDIATRIC PATIENTS IN CROATIA: DATA FROM THE CROATIAN NATIONAL REGISTRY}

Lana Ivković* ${ }^{*}$ Iva Hojsak, Ivana Trivić, Sara Sila, Pero Hrabač, Vlatka Konjik, Irena SenečićČala, Goran Palčevski, Ranka Despot, Orjena Žaja, Sanja Kolaček. Children's Hospital Srebrnjak, Zagreb, Croatia

10.1136/archdischild-2021-europaediatrics.229

Aims of this study were to determine the phenotype of pediatric inflammatory bowel disease (IBD) in patients in Croatia at the time of disease presentation, evaluate diagnostic and therapeutic approaches and investigate early disease-course in oneyear follow-up.

During a one-year time period (June 1st, 2016 to May 31st, 2017), children and adolescents younger than 18 years of age in Croatia with newly diagnosed IBD were prospectively recruited into Croatian national registry. Data on disease location and behavior (according to Paris classification), diagnostic evaluation and therapy used for inducing and maintaining remission were collected. One-year follow-up data examined relapse rates, as well as modification of medical therapy.

A total of 51 newly diagnosed patients were recruited: 19 (37.3\%) Crohn's disease (CD), 28 (54.9\%) ulcerative colitis (UC) and 4 (7.8\%) IBD - unclassified (IBD-U). Most common location in $\mathrm{CD}$ was ileocolonic (L3) disease in $52.6 \%$, followed by distal $1 / 3$ ileal disease (L1) in 31.6\%. 